\title{
Measuring the orbital angular momentum density for a superposition of Bessel beams
}

\author{
A. Dudley ${ }^{\mathrm{a}, \mathrm{b}}$, I. Litvin ${ }^{\mathrm{a}}$ and A. Forbes ${ }^{*, \mathrm{a}, \mathrm{b}}$ \\ ${ }^{a}$ CSIR National Laser Centre, PO Box 395, Pretoria 0001, South Africa; \\ ${ }^{b}$ School of Physics, University of KwaZulu-Natal, Private Bag X54001, Durban 4000, South Africa
}

\begin{abstract}
In this report we theoretically calculate and experimentally measure the OAM density of a coherent superposition of both symmetric and non-symmetric non-diffracting Bessel beams. Although the intensity pattern of the superimposed field rotates at a fixed angular velocity (which is due to the differing wave-vectors of the component fields), we show that the magnitude and direction of the OAM is dependent on the radial position within the field. We outline a simple approach using only a spatial light modulator to measure the OAM density for a superposition of non-diffracting Bessel beams. Our quantitative measurements are in good agreement with predicted values.
\end{abstract}

Keywords: orbital angular momentum density, spatial light modulator, superpositions of non-diffracting Bessel beams

\section{INTRODUCTION}

Fields which carry OAM of $l \hbar$ per photon, some of which include Laguerre-Gaussian beams [1], Bessel-Gaussian beams [2] and Airy beams [3], have an azimuthal angular dependence of $\exp (i l \varphi)[1,4]$, where $l$ is the unbounded azimuthal mode index and $\varphi$ is the azimuthal angle. Since the discovery of light beams carrying orbital angular momentum (OAM) [4], many techniques for the measurement of OAM have been developed, ranging from interferometers [5-7] to computer generated holograms [8-11]. These aforementioned techniques do not allow one to obtain a quantitative measurement for the OAM density, and instead only measure the average OAM across the entire field.

In this report we theoretically calculate and experimentally measure the OAM density of both symmetric and non-symmetric superpositions of non-diffracting Bessel beams $[12,13]$. We outline a simple approach using only a spatial light modulator to measure the OAM density for a superposition of non-diffracting Bessel beams. The ability to measure the OAM distribution of optical fields has relevance in optical tweezing, and quantum information and processing.

\section{THEORY}

\section{Symmetric superposition}

We will first start with the symmetric case and later develop our argument for the theoretical and experimental OAM densities for the non-symmetric case. The amplitude for a symmetric superposition of two Bessel beams, of opposite azimuthal order, is given by

$$
u(r, \theta, z)=A_{0}\left(J_{l}\left(q_{1} r\right) \exp (i \Delta k z) \exp (i l \theta)+\alpha_{0} J_{-l}\left(q_{2} r\right) \exp (-i \Delta k z) \exp (-i l \theta)\right),
$$

where $J_{l}$ is the Bessel function of order $l . q_{1}$ and $q_{2}$ are the radial wave numbers, $\Delta k$ is the difference between the two longitudinal wave numbers, $\alpha_{0}$ is the ratio between the two component Bessel fields, and $A_{0}$ is a normalization constant.

*aforbes1@csir.co.za

Complex Light and Optical Forces VI, edited by Enrique J. Galvez, David L. Andrews, Jesper Glückstad

Marat S. Soskin, Proc. of SPIE Vol. 8274, 827406 - C 2012 SPIE · CCC code: 0277-786X/12/\$18 · doi: 10.1117/12.910779 
The following result for the Poynting vector

$$
\vec{S}=\varepsilon_{0} c^{2}\left\langle\vec{E}_{\text {real }} \times \vec{B}_{\text {real }}\right\rangle=\frac{i \omega \varepsilon_{0}}{4}\left(u \nabla u^{*}-u^{*} \nabla u\right)+\varepsilon_{0} \omega k|u|^{2} \vec{z},
$$

along with the total OAM density (in the direction of propagation, $z$ )

$$
L_{z}=\frac{1}{c^{2}}(r \times S)_{z},
$$

results in the total OAM density for the field given in Eq. (1) to be

$$
L_{z}(r)=\frac{-A_{0}^{2} l \varepsilon_{0} \omega}{2 c^{2}}\left(J_{l}^{2}\left(k_{r 1} r\right)-\alpha_{0}^{2} J_{-l}^{2}\left(k_{r 2} r\right)\right)
$$

The measurement of the OAM density is achieved by performing a modal decomposition of the optical field. It is executed by obtaining the inner product of the incoming field with a predetermined match filter, which is programmed on to a spatial light modulator, for various OAM values and at particular radial positions. The weighting of each azimuthal mode is determined by the following inner product

$$
a_{l}(r, z)=\frac{1}{\sqrt{2 \pi}} \int_{0}^{2 \pi} u(r, \phi, z) \exp (-i l \phi) d \phi
$$

allowing the OAM density to be experimentally measured by the following relation

$$
L_{z}(r)=\frac{l \omega}{2 c \eta}\left(\frac{\lambda f}{S_{\text {Ring }}}\right)^{2}\left(I_{l}(0)-I_{-l}(0)\right) .
$$

$\eta$ is a factor for the optical efficiency of the experimental setup and $S_{\text {Ring }}$ is the area $(4 \pi \Delta R . R)$ of the ring-slit in the match-filter and is a function of its radial position, $R$.

The optical field in Eq. (1) can be extended to a generalized form

$$
u(r, \theta, z)=A_{0} \sum_{l=-N}^{N} \alpha_{l} J_{l}\left(q_{l} r\right) \exp \left(i \Delta k_{l} z\right) \exp (i l \theta)+\alpha_{-l} J_{-l}\left(q_{-l} r\right) \exp \left(-i \Delta k_{-l} z\right) \exp (-i l \theta)
$$

where $\alpha_{l}$ and $\alpha_{-l}$ denote the energy contained in each of the ring-slits, with respects to the energy contained in the innermost ring-slit. Due to the fact that the $\varphi$-component of the Poynting vector, $S_{\varphi}$, is a linear operator, the OAM density for the generalized field described in Eq. (4) can be described both theoretically and experimentally by extending the results given in Eqs (4) and (6) as

$$
L_{z}(r)=\frac{\varepsilon_{0} \omega A_{0}^{2}}{2} \sum_{l=-N}^{N} l \alpha_{l}^{2} J_{l}^{2}\left(q_{l} r\right)-l \alpha_{-l}^{2} J_{-l}^{2}\left(q_{-l} r\right)
$$


and

$$
L_{z}(R)=\frac{\omega}{2 c \eta}\left(\frac{\lambda f}{S_{\text {Ring }}}\right)^{2} \sum_{l=-N}^{N} l\left(I_{l}(0)-I_{-l}(0)\right)
$$

respectively.

\section{Non-symmetric superposition}

The amplitude of a non-symmetric superposition of two Bessel beams is described as

$$
u(r, \theta, z)=A_{0}\left(J_{l}\left(q_{1} r\right) \exp (i \Delta k z) \exp (i l \theta)+\alpha_{0} J_{m}\left(q_{2} r\right) \exp (-i \Delta k z) \exp (i m \theta)\right),
$$

where the azimuthal indices are of different orders, $l \neq m$. The OAM density is determined theoretically by following the same procedure in Eqs (2) and (3),

$$
L_{z}(r, \phi, z)=\frac{\varepsilon_{0} \omega A_{0}^{2}}{2}\left(l J_{l}^{2}\left(q_{1} r\right)+m \alpha_{0}^{2} J_{m}^{2}\left(q_{2} r\right)+(l+m) \alpha_{0} \cos ((l-m) \phi+2 \Delta k z) J_{l}\left(q_{1} r\right) J_{m}\left(q_{2} r\right)\right) .
$$

Equation 11 is evaluated by summing over the OAM density values for a range of angles varying from 0 to $2 \pi$. The term $\Delta k z$ can be ignored as its effect on the optical field is only a constant phase-shift.

The quantifiable measurement to the theoretical OAM density is determined as [13]

$$
L_{z}(r, \phi, z)=\frac{\omega}{2 c \eta}\left(\frac{\lambda f}{S_{\text {Ring }}}\right)^{2}\left(l I_{l}(0)+m I_{m}(0)+(l+m) \cos ((l-m) \phi+2 \Delta k z) \sqrt{I_{l}(0)} \sqrt{I_{m}(0)}\right) .
$$

The experimental equation for the OAM density is integrated over $\varphi$, resulting in the average OAM density

$$
\overline{L_{z}(r)}=\frac{\omega}{2 c \eta}\left(\frac{\lambda f}{S_{\text {Ring }}}\right)^{2}\left(l I_{l}(0)+m I_{m}(0)\right)
$$

where the term $\Delta k z$ can be neglected.

The amplitude of the non-symmetric superposition can be extended to consist of many Bessel beams

$$
u(r, \theta, z)=A_{0} \sum_{l=-N}^{N} \sum_{m=-N}^{N} \alpha_{l} J_{l}\left(q_{l} r\right) \exp \left(i \Delta k_{l} z\right) \exp (i l \theta)+\alpha_{m} J_{m}\left(q_{m} r\right) \exp \left(i \Delta k_{m} z\right) \exp (i m \theta) .
$$

Similarly the $\varphi$-component of the Poynting vector, $S_{\varphi}$, is a linear operator and the theoretical and experimental OAM densities can be extracted by extending the simple forms in Eqs (11) and (13), however since the equations become very cumbersome they have been neglected in this manuscript.

\section{EXPERIMENTAL METHODOLOGY}

To execute the modal decomposition given in Eq. (5), an inner product of the incoming field with a match filter, $\exp (\mathrm{i} l \varphi)$, was performed and is denoted in Fig. 1. A HeNe laser $(\lambda \sim 633 \mathrm{~nm})$ was expanded through a $6 \times$ telescope and directed onto the liquid crystal display (LCD) of a SLM. The first SLM (denoted as LCD 1) was programmed to produce various superposition fields using the concept of Durnin's ring-slit [14], but implemented digitally [15]. 
The resulting images of the symmetric and non-symmetric non-diffracting superposition field were captured on a CCD camera. The resulting superposition field was then magnified with a $10 \times$ objective and directed to the second SLM (LCD 2) for executing the modal decomposition of Eq. (5).

The incoming field was divided into 10 annular rings and the phase within each annular ring varied as the complex conjugate of the azimuthal modes present in the incoming superposition field, from $l=-4$ to 4 . Since the weighting coefficients, $\left(a_{l}(r)\right)^{2}$, can be experimentally measured as a function of the radial co-ordinate and the azimuthal mode, by measuring the on-axis intensity of the inner-product, the OAM density can be quantitatively measured.

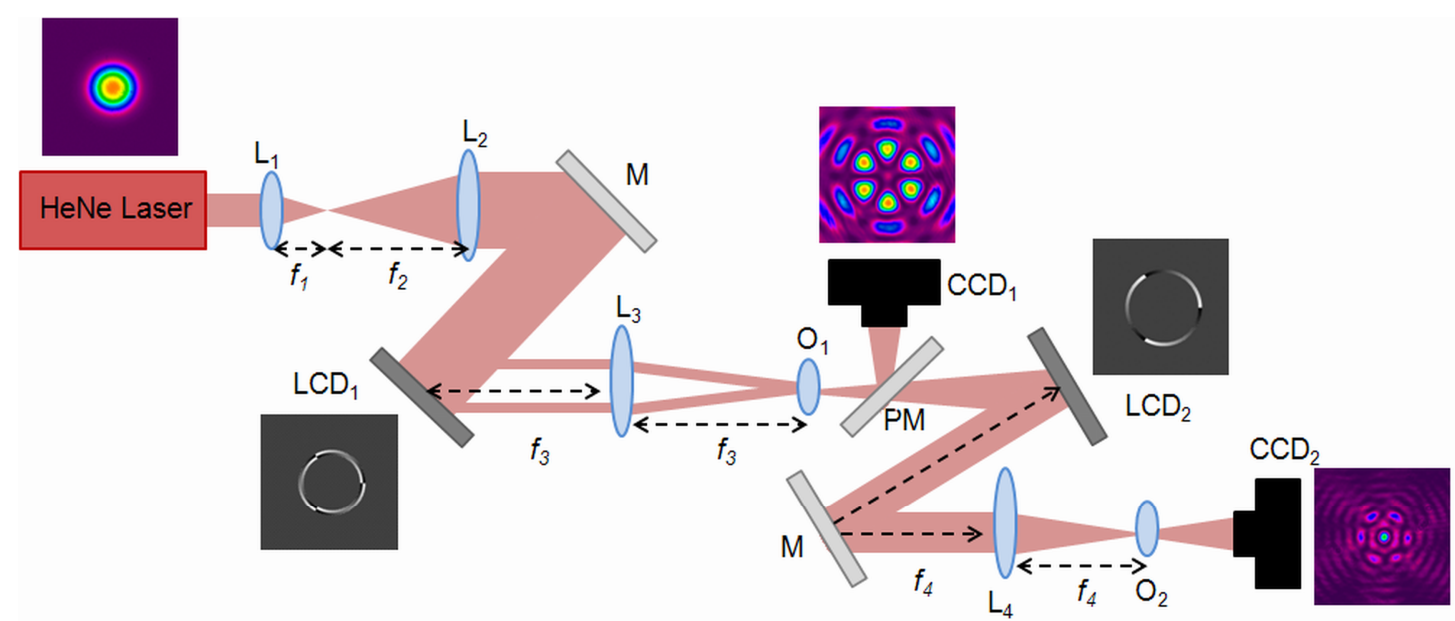

Fig. 1. A schematic of the experimental setup for measuring the OAM density of symmetric and non-symmetric superpositions of Bessel beams as a function of the radial position, $R$. L: Lens $\left(f_{1}=25 \mathrm{~mm} ; \mathrm{f}_{2}=150 \mathrm{~mm} ; \mathrm{f}_{3}=200 \mathrm{~mm}\right.$ and $\mathrm{f}_{4}=200 \mathrm{~mm}$ ); M: Mirror; LCD: Liquid Crystal Display; O: Objective; PM: Pop-up Mirror; CCD: CCD Camera. The objective, $\mathrm{O} 2$, was placed at the focus (or Fourier plane) of lens, $\mathrm{L}_{4}$. The corresponding optical fields or holograms are represented at the appropriate planes.

\section{RESULTS AND DISCUSSION}

\section{Symmetric superposition}

The first digital hologram consists of a ring-slit separated into two ring-slits (of equal widths), each possessing an azimuthal phase of equal order but opposite handedness, (i.e. $l_{\text {inner }}=3$ and $l_{\text {outer }}=-3$ ). The dimensions (in pixels) of the ring-slit are: $r_{1}=173, r_{2}=188, \Delta r_{1}, \Delta r_{2}=15$. In the case that $\mathrm{LCD}_{1}$ was encoded with two ring-slits, where the orders of the two azimuthal phases are of equal but opposite handedness, a 'petal'-structure was produced, where the number of 'petals' is denoted by $2|l|$ as expected from theory [15].

Since the match-filter is programmed digitally, the radius of the ring-slit, as well as the azimuthal phase within the ring-slit can be easily varied. This dynamical aspect of the SLM allows us to radially locate where in the optical field we wish to make a measurement of the OAM density. In this paper, the match-filters consist of ring-slits having ten different radii $\left(r_{1}=75 ; r_{2}=110 ; r_{3}=145 ; r_{4}=180 ; r_{5}=215 ; r_{6}=250 ; r_{7}=285 ; r_{8}=320 ; r_{9}=355\right.$ and $r_{10}=390$ (given in pixels), each consisting of a width of 20 pixels), where the phase within the rings, $n$, varied as the complex conjugate of the azimuthal modes present in the incoming optical field. The OAM density for a particular radial position can then be measured directly from Eqs (4) and (6) by measuring the on-axis intensity of the inner-product. The measured OAM densities as a function of the radial position for the symmetric optical field are given in Fig. 2. 

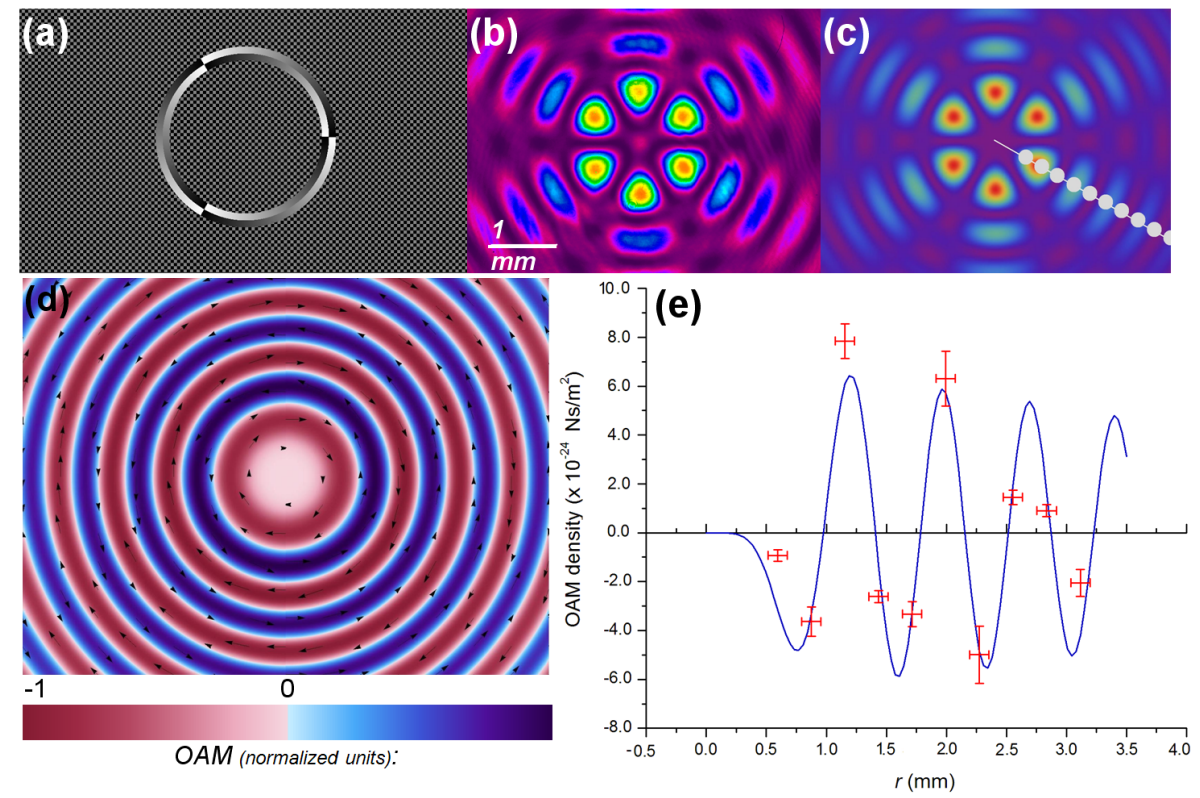

Fig. 2. (a) The digital ring-slit and the corresponding experimentally produced field in the Fourier plane (b) accompanied with theoretically calculated fields (c). The ten white dots in (c) denote the radial positions of each of the ten ring-slits used in the match-filters. (d) A density plot of the OAM density for the field given in (b). Red denotes negative OAM and blue denotes positive. Light to dark blue denotes an increase in positive OAM and light to dark red denotes an increase in negative OAM. (e) the theoretical (blue curve) and experimentally measured (red points) OAM density.

Since the incoming optical field consists of an equal weighting of $l_{\text {inner }}=3$ and $l_{\text {outer }}=-3$ order Bessel beams, the OAM density represented in the $x-y$ plane consists of evenly-sized concentric rings of positive and negative OAM. This is also evident in the radial cross-sectional profile of the OAM density where the OAM density oscillates evenly around a value of zero.

\section{Non-symmetric superposition}

The second digital hologram was divided into three ring-slits, having the following azimuthal phase variations $l_{\text {inner }}=-3$, $l_{\text {middle }}=2, l_{\text {outer }}=1$, producing a non-symmetric superposition. The dimensions (in pixels) of the ring-slits are: $r_{1}=170$, $r_{2}=180, r_{3}=190, \Delta r_{1}, \Delta r_{2}, \Delta r_{3}=10$. Our experimental field (Fig. 3 (b)) is in very good agreement with the theoretically calculated field (Fig. 3 (c)). Since the ring-slits are equally weighted and the azimuthal mode indices of the ring-slits, in Fig. 3 (a), sum to zero, the global OAM is zero, giving rise to the OAM density existing equally in both the negative and positive quadrants for the OAM density across the radial direction of the field, evident in Fig. 3 (d) and (e). The OAM density is not uniform in all radial directions of the optical field (evident in Fig. 3 (e)) and so the OAM density plotted in Fig. 3 (d) is an average over all angular positions. 

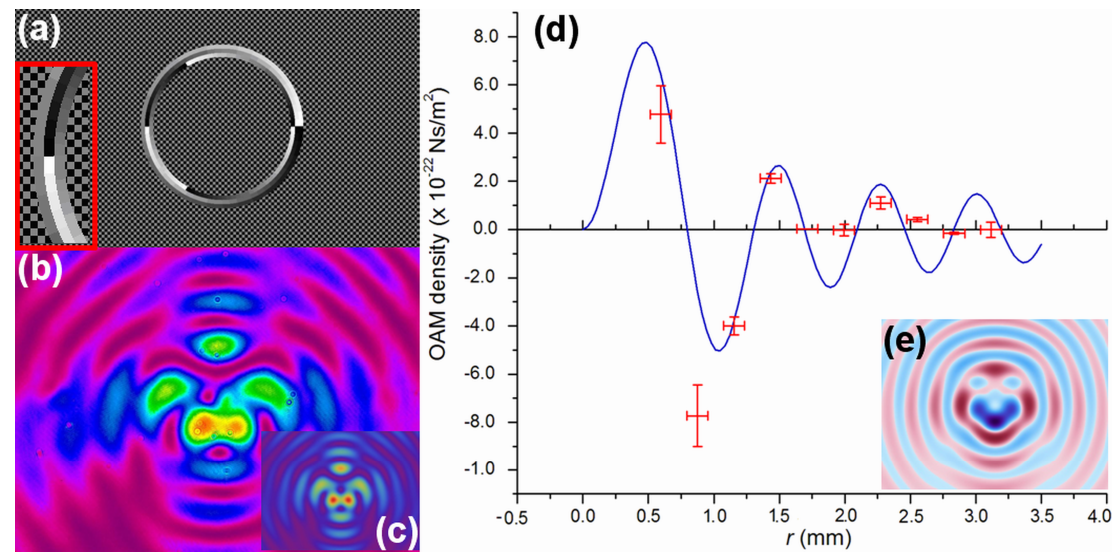

Fig. 3. (a) The digital hologram used to generate the experimental field given in (b) of which the theoretical field is represented in (c). A magnification of the ring-slit is given as an insert in (a). (d) The theoretical (blue curve) and experimentally measured (red points) OAM density. (e) A density plot of the OAM density. Red denotes negative OAM and blue denotes positive. Light to dark blue denotes an increase in positive OAM and light to dark red denotes an increase in negative OAM.

\section{CONCLUSION}

We have used the Poynting vector approach to derive expressions for the OAM density for symmetric and nonsymmetric optical fields theoretically as well as experimentally. We obtain quantitative measurements for the OAM density as a function of the radial position for both a symmetric and a non-symmetric superposition of non-diffracting Bessel beams, illustrating good agreement with the theoretical prediction. We find that while the global OAM is zero, the local OAM spectrum changes radially across the beam, and can be made to oscillate from positive to negative values by a suitable choice of the parameters making up the superposition, making it an ideal tool in the field of optical trapping and tweezing.

\section{REFERENCES}

[1] Beijersbergen, M. W., Allen, L., van der Veen, H. E. L. O. and Woerdman, J. P., "Astigmatic laser mode converters and the transfer of orbital angular momentum," Opt. Commun. 96(1-3), 123-132 (1993).

[2] Arlt, J. and Dholakia, K., "Generation of high-order Bessel beams by use of an axicon," Opt. Commun. 177(16), 297-301 (2000).

[3] Sztul, H. I. and Alfano, R. R., "The Poynting vector and angular momentum of Airy beams," Opt. Express 16(13), 9411-9416 (2008).

[4] Allen, L., Beijersbergen, M. W., Spreeuw R. J. C. and Woerdman, J. P., "Orbital angular momentum of light and the transformation of Laguerre-Gaussian laser modes," Phys. Rev. A 45(11), 8185-8189 (1992).

[5] Leach, J., Padgett, M. J., Barnett, S. M., Franke-Arnold, S. and Courtial, J., "Measuring the orbital angular momentum of a single photon," Phys. Rev. Lett. 88(25), 257901 (2002).

[6] Lavery, M., Dudley, A., Forbes, A., Courtial, J. and Padgett, M., "Robust interferometer for the routing of light beams carrying orbital angular momentum," NJP 13, 093014 (2011).

[7] Gao, C., Qi, X., Liu, Y., Xin, J. and Wang, L., "Sorting and detecting orbital angular momentum states by using a Dove prism embedded Mach-Zehnder interferometer and amplitude gratings," Opt. Commun. 284(1), 48-51 (2011).

[8] Mair, A., Vaziri, A., Weihs, G., and Zeilinger, A., "Entanglement of the orbital angular momentum states of photons," Nature 412(6844), 313-316 (2001).

[9] Gibson, G., Courtial, J., Padgett, M. J., Vasnetsov, M., Pas'ko, V., Barnett, S. M. and Franke-Arnold, S., "Freespace information transfer using light beams carrying orbital angular momentum," Opt. Express 12, 5448-5456 (2004). 
[10] Khonina, S. N., Kotlyar, V. V., Skidanov, R. V., Soifer, V. A., Laakkonen, P. and Turunen, J., "Gauss-Laguerre modes with different indices in prescribed diffraction orders of a diffractive phase element," Opt. Commun. $175,301(2000)$.

[11] Berkhout, G. C. G., Lavery, M. P. J., Courtial, J., Beijersbergen, M. W. and Padgett, M. J., "Efficient Sorting of Orbital Angular Momentum States of Light," Phys. Rev. Lett. 105, 153601 (2010).

[12]Litvin, I., Dudley, A. and Forbes, A., "Poynting vector and orbital angular momentum density of superpositions of Bessel beams," Opt. Express 19(18), 16760-16771.

[13] Dudley, A., Litvin, I. and Forbes, A., "Quantitative measurement of the orbital angular momentum density of light," - accepted by Applied Optics.

[14] Durnin, J., "Diffraction-free beams," Phys. Rev. Lett. 58, 1499-1501 (1987).

[15] Vasilyeu, R., Dudley, A., Khilo, N. and Forbes, A., "Generating superpositions of higher-order Bessel beams," Opt. Express 17, 23389-23395 (2009). 